\title{
Tecnologias aplicadas em sanidade de peixes
}

\section{Henrique César Pereira Figueiredo, Carlos Augusto Gomes Leal}

Médico Veterinário, Professor do Departamento de Medicina Veterinária da Universidade Federal de Lavras Coordenador do AQUAVET - Lab. de Doenças de Animais Aquáticos

Médico Veterinário, Mestrando em Ciências Veterinárias - Universidade Federal de Lavras

A aqüicultura é o setor da agropecuária brasileira com maior expansão nos últimos anos, que segundo dados do último levantamento da Food and Agriculture Organization (FAO, 2006) o crescimento da atividade no Brasil superou a média mundial. Essa é apontada como um mercado estratégico para o desenvolvimento sustentável, produção de alimentos e ampliação de fronteiras inesploradas no país. Apesar da importância do setor, as atividades de aqüicultura ainda não foram incluídas no censo agropecuário brasileiro. Em face à necessidade premente de informações estatísticas sobre a aqüicultura no país, o Ministério da Ciência e Tecnologia e o Conselho Nacional de Desenvolvimento Científico e Tecnológico articularam um levantamento em âmbito nacional das características, perspectivas e limitações da atividade aquícola (Valenti et al, 2000). Os resultados desse estudo mostraram as aptidões atuais e perspectivas de cada região do país, bem como os entraves para o aumento da produção e da produtividade dos plantéis. Dentre os entraves apontados, os aspectos sanitários da produção e a falta de estrutura para o diagnóstico das principais enfermidades infecciosas foram considerados de grande relevância.

Dentre as bactérias potencialmente patogênicas para peixes destacam-se as Aeromonas móveis, Flavobacterium columnare e Streptococcus agalactiae. Em Minas Gerais e no Brasil o controle dessas doenças têm sido feito somente pelo uso de antibióticos incorporados à ração (para animais em engorda), ou administrados diretamente na água de cultivo para tratamento de alevinos. Em ambientes aquáticos, essas drogas ocasionam grande impacto no ambiente, associado a resíduos químicos na água, bem como a seleção de bactérias resistentes. Ainda, nos últimos anos, os resíduos de antibióticos em carcaças de peixes têm sido uma barreira ao comércio internacional, principalmente o destinado à exportação para os Estados Unidos e Comunidade Européia.

Avanços cientificos recentes, principalmente no campo da biotecnológica, tem auxiliado no esclarecimento e controle dos processos patogênicos em peixes. Ferramentas e técnicas de biologia molecular têm sido desenvolvidas e utilizadas para o diagnóstico das principais doenças, identificação de fatores de virulencia, caracterização molecular de isolados e desenvolvimento de vacinas. A seguir serão discutidos as principais tecnologias e avanços nesses campos.

\section{Patógenos Emergentes na Piscicultura}

As infecções por Aeromonas móveis são de ocorrência mundial e provavelmente a doença bacteriana mais comum em peixes de água doce (Noga, 1996). Essas possuem como fatores predisponentes diversas condições de estresse as quais podem ser submetidas as populações de peixes, como o excesso de matéria orgânica na água, oxigênio dissolvido abaixo das concentrações adequadas e alta densidade animal, no entanto. Descrição mais antigas consideram esses patógenos como oportunistas, atualmente essas bactérias foram relatadas como emergentes agentes primários, possuindo mecanismos altamente específicos para promoção de doenças (Chacon et al., 2004; Vilches et al., 2004; Sha et al., 2005; Yu et al., 2005)O gênero Aeromonas compreende um grupo de organismos que têm amplamente distribuidas em ambientes aquáticos. Adaptadas ao crescimento em temperaturas que variam de $5^{\circ} \mathrm{C}$ a $37^{\circ} \mathrm{C}$, essas são bastonetes Gram-negativos, oxidase positivos, anaeróbicos facultativos e estão presentes na superfície e brânquias dos peixes 
(Kozinska, 2007). A taxonomia deste grupo sofreu muitas alterações nas últimas décadas e até o presente momento a caracterização das especies por testes bioquímicos e métodos moleculares não está totalmente padronizada, havendo ainda muitas controversias (Saadreva et al., 2006). Aeromonas caviae, Aeromonas sobria, Aeromonas hydrophila, Aeromonas bestiarum e Aeromonas veronii são as principais causadoras de doenças em peixes, que em conjunto são denominadas de "septicemia por aeromonas moveis". Essas são chamadas de móveis pois apresentam motilidade por flagelos polares e laterais. Os sinais clínicos de infecção podem variar de lesões de pele, superficiais ou profundas, a quadros típicos de septicemia. As lesões de pele podem se apresentar como áreas de hemorragia $\mathrm{e}$ necrose de extensão variada, que podem progredir para úlceras que acometem geralmente o tecido muscular. Nos quadros de infecção sistêmica são observados a exoftalmia, abdômen distendido contendo líquido serosanguinolento e presença de petéquias hemorrágicas nas vísceras (Pavanelli et al, 2002).

Os fatores de virulência da bactéria estão relacionados com a invasão, replicação e evasão do sistema imune hospedeiros, alem de provocarem as lesões durante a patogênese da doença (Vilches et al., 2004). Vários fatores de virulência têm sido descritos em Aeromonas hydrophila. Dentre eles antígeno O, cápsulas, a camada $\mathrm{S}$, exotoxinas como hemolisinas e enterotoxinas, exoenzimas como lipases, amilases e proteases e mais recentemente o sistema de secreção tipo III (SSTT) (Yu et al., 2004).

A columnariose é uma doença de distribuição mundial que acomete a maioria das espécies de peixes de água doce. Altamente impactante, é a segunda enfermidade que mais causa prejuízos à indústria americana do "catfish" (Arias, et al., 2004). No Brasil não existem dados sobre seu impacto na produção de peixes, porém, surtos da doença são frequentemente observados nas pisciculturas nacionais, principalmente nas larviculturas. Flavobacterium columnare é o agente etiológico dessa doença. Fenotipicamente essa bactéria é caracterizada como um bastonete longo, Gram negativo, móvel, forma colônias filamentosas de coloração amarelada (produz um pigmento denominado flexirubina) e não cresce em meios de cultura convencionais. A sintomatologia clinica da doença é caracterizada pela erosão da pele e nadadeiras. Com a evolução do quadro (24-48 horas após) ocorre o acometimento de tecidos adjacentes, causando necrose muscular e destruição total das nadadeiras (Decostere, et al, 1999).

A ocorrência dessa doença está associada a fatores predisponentes que aumentam a susceptibilidade dos animais ao agente etiológico. Amostras com alta virulência podem infectar peixes sadios, porém, o estresse oriundo da manipulação grosseira, transporte prolongado, baixa qualidade de água, lesões mecânicas etc. colaboram e propiciam o desencadeamento de surtos (Suomalainen et al., 2005a). Adesinas e a enzima condroitina AC-liase são os principais fatores de virulência associados às infecções por $F$. columnare. A aderência às superfícies corpóreas (pele, brânquias $\mathrm{e}$ nadadeiras) é uma etapa fundamental na patogênese da doença. A virulência das amostras está diretamente relacionada à sua capacidade de aderir à superfície corporal do hospedeiro (Decostere, et al, 1999). Uma correlação entre a de infestações parasitarias na pele e brânquias e o aumento na infectividade das amostras $F$. columnare tem sido observado na prática. Trutas arcoíris parasitadas por digenéticos são mais susceptíveis a columnariose quando infectadas experimentalmente (Suomalainen et al., 2005b).

As bactérias do gênero Streptococcus são patogênicas para uma ampla gama de hospedeiros, dentre eles seres humanos, peixes de água doce e marinhos. As infecções causadas por esses microrganismos são impactantes para diversos segmentos da produção animal, incluindo a aqüicultura. Duas espécies têm sido descritas como os principais agentes etiológicos causadores de infecções nervosas em peixes: Streptococcus agalactiae e Streptococcus iniae. Esses ocasionam enormes prejuízos principalmente para a tilapicultura mundial (Evans et al., 2000; Agnew e Barnes, 2007; Mian et al., in press). Infecções causadas Streptococcus agalactiae vem sendo descritas na literatura desde meados do século 30. Também conhecido com Streptococcus do Grupo B, essa bactéria é um importante patógeno para seres humanos estando associada a casos de meningoencefalites em recém-nascidos (Maione et al., 2005). Em bovinos, esse microrganismo é responsável por quadros de mamite clínica e subclínica (Keefe, 1997). Nas ultimas décadas essa bactéria tem se 
destacado como patógeno emergente na piscicultura. Casos de infecção por essa bactéria foram descritas em mais de 20 espécies de peixes (Olivares-Fuster et al., 2008). No Brasil, o primeiro relato de infecção por $S$. agalactiae em peixes foi realizada no ano de 2006 por Figueiredo et al.

Streptococcus iniae é um patógeno que acomete diversas espécies de peixes e atualmente têm sido descrito como zoonótico para humanos. O primeiro isolamento dessa bactéria ocorreu na década de 70 , a partir de abscessos subcutâneos de um golfinho. Desde então, surtos de doenças em piscicultura, causadas por esse microrganismo, foram observadas na maioria dos continentes. Atualmente, essa bactéria é um dos patógenos de maior relevância para a produção de peixes, principalmente na Ásia (Agnew e Barnes, 2007). No Brasil até o presente momento não existem relatos do isolamento e identificação dessa bactéria.

A introdução desses agentes em uma propriedade livre ocasiona um impacto devastador na criação, com morbidade e mortalidade elevadas, que pode dizimar o plantel (Panisk et al, 2005). Os fatores predisponentes são os mesmos das demais doenças bacterianas em peixes (estresse, variação brusca na temperatura da água, excesso de amônia, alta densidade de estocagem, baixa qualidade de água etc.) (Panisk et al., 2005). As infecções por S. agalactiae em peixes são caracterizadas por quadros de septicemia e meningoencefalite. Já as causadas por $S$. iniae manifestam-se de forma diferente de acordo com o sorotipo envolvido. A doença pode ocorrer na forma de abscessos multifocais na musculatura dos animais, denominada de "gulf ball disease" ou de maneira clássica (septicêmicos e meningoencefalites). Os principais sinais clínicos são abscessos multifocais ("gulf ball disease"), natação errática e em rodopios, exoftalmia unilateral ou bilateral, perda de escamas, escurecimento do tegumento, anorexia, excitabilidade e morte. Após alta mortalidade inicial, a doença atinge um quadro endêmico no plantel. Caso o tratamento não seja realizado essa pode persistir na propriedade através dos sucessivos ciclos de produção (Agnew e Barnes, 2007).

\section{Diagnóstico, Fatores Virulência e Caracterização Molecular dos Isolados}

As técnicas de biologia molecular propiciaram uma melhora significativa na eficiência e qualidade do diagnóstico de enfermidades de peixes. Isso ocorreu principalmente para doenças causadas por vírus $\mathrm{e}$ bactérias de difícil isolamento e cultivo em laboratório. Inicialmente técnicas de hibridização eram utilizadas para detectar e comparar porções do genoma dos microrganismos. Porém, na década de 80 foi desenvolvida a reação em cadeia da polimerase (PCR sigla em inglês). Essa técnica permite a clonagem "in vitro" de segmentos específicos do DNA patógenos, detectados por meio de pequenas sondas chamadas de primers. Esses ligam-se a seqüências especifica e são reconhecidas pela enzima DNA polimerase que inicia a produção de uma seqüência complementar a fita molde. Posteriormente os produtos são visualizados por eletroforese em gel de agarose. Devido à especificidade dos primers e agilidade a PCR é ferramenta molecular (sem sombra de dúvida) mais utilizada para o diagnóstico de doenças em animais, seres humanos e plantas. Outra aplicação importante dessa técnica e para a detecção de genes de virulência nos isolados, permitindo inferência sobre a patogenicidade das diferentes amostras.

O SSTT é um importante fator de virulência para diversas espécies de bactérias Gram negativas e positivas (Cornelis et al., 2000). O estudo desse fator de virulência pela técnica de PCR tem um interesse especial devido à intrínseca relação que promove entre as células da bactéria e do hospedeiro. Esse sistema é composto por uma nano-maquinária microscópica em formato de agulha e proteínas efetoras da bactéria que são injetadas diretamente na célula hospedeira, alterando funções fisiológicas e a homeostasia (Coburn et al., 2007). Grande parte da virulência de amostras de Aeromonas hydrophila tem sido atribuída à presença do SSTT, visto a disparidade na virulência de isolados positivos e negativos para esses fator de virulência, sendo os isolados negativos significativamente menos patogênicos que os positivos.

Devido à ampla distribuição mundial da $F$. columnare, a variabilidade genética e fenotipica entre amostras de diferentes origens geográficas têm sido descritas na literatura. Isolados brasileiros de $F$. columnare analisados pela técnica de FAME mostraram-se diferentes de norte-americanos e israelenses, com possível implicação no padrão antigênico das diferentes amostras (Figueiredo et al., 2005). O diagnóstico é realizado pelo isolamento da 
bactéria em meios de cultura especiais e posteriormente a identificação por técnicas bioquímicas ou moleculares (Bader e Shotts, 1998; Bader et al., 2003; Darwish et al., 2004). Dentre as técnicas moleculares PCR é sem duvida a ferramenta mais eficiente e utilizada para o diagnóstico da columnariose. Diversos protocolos são utilizados, geralmente com primers específicos para regiões conservadas do genoma das bactérias (Bader et al., 2003; Darwish et al, 2004). Vacinas sintetizadas com isolados específicos têm sido utilizadas para a prevenção da columnariose (Bader et al., 1997; Grabowski et al, 2002), contudo, a existência de proteção cruzada entre amostras de diferentes regiões não foi determinada.

Diversas técnicas moleculares tem sido utilizados para a caracterização molecular de S. agalactiae, dentre elas a eletroforese em campo pulsado (PFGE) e o sistema de tipificação de multi lócus (MLST). A PFGE requer a utilização de uma única enzima de restrição que possua sítio pouco freqüente no DNA, o que resulta em um número reduzido de fragmentos grandes, facilitando sua visualização, e tem boa reprodutibilidade. Portanto, os perfis eletroforéticos obtidos para as diferentes cepas são mais facilmente comparados em relação aos obtidos com técnicas de eletroforese convencionais (Fasola, Livdahl e Ferrieri, 1993). A técnica de MLST é baseada na amplificação e sequenciamento de no mínimo três genes conservados, o que permite realizar inferências sobre proximidade evolutiva dos diferentes isolados. Essa técnica tem sido para diversos estudos em GBS isolados de seres humanos, principalmente para determinação de diferentes sorotipos (Martins et al., 2007).

\section{Desenvolvimento de Vacinas}

Em todo mundo o uso de vacinas como método imunoprofilático na aqüicultura tem aumentado significativamente, seguindo a tendência de expansão da atividade. Porem, a vacinologia em peixes é ainda uma área prematura, tanto cientificamente como na capacidade de transferência de tecnologia para as indústrias (Gudding et al., 1999). Vacinas contra os principais patógenos para peixes têm sido desenvolvidas (Gudding et al., 1999; Klesius et al., 2004; Vanderberg, 2004; Agnew e Barnes, 2007; Tonheim et al., 2008). Os peixes podem ser imunizados por via injetável, preferencialmente por injeção intraperitonial (i.p.); por imersão, onde os animais são imersos em solução aquosa contendo os antígenos vacinais; ou pela administração oral das vacinas. Esses métodos apresentam diferentes vantagens $\mathrm{e}$ desvantagens quanto à eficiência da imunização, praticidade, efeitos colaterais e custo - benefício (Gudding et al., 1999). O método de administração afeta diretamente a imunidade protetora induzida pela vacinação. As vacinas injetáveis são as que induzem respostas inatas e adaptativas em maior intensidade e mais duradouras, em comparação as demais vias. Porem, na prática vacinas de baixo custo e que não demandem manejo intenso dos animais durante a vacinação são as que apresentam melhores resultados em pisciculturas tropicais. Métodos não injetáveis, como a imersão, permitem a imunização de milhares de peixes em um período reduzido de tempo (Klesius et al., 2004), contudo, grandes quantidades de vacina são necessárias (Vanderberg, 2004). Em virtude da praticidade do processo e ausência de estresse, as vacinas orais têm se tornado uma alternativa viável para imunização dos peixes. Apesar disso, os níveis de resposta atingidos por essa via quando utilizada como única forma de imunização são baixos (Romalde et al., 2004) e até casos de imunossupressão podem ser observados (Vanderberg, 2004). Resultados positivos têm sido obtidos com o uso de veículos orais a reimunização dos peixes. Romalde et al. (2004) avaliaram o uso de micropartículas de alginato como veiculo oral para a imunização de peixes, verificando seu efeito como método único de vacinação e como reforço ("booster") para doses primárias via i.p. ou imersão. A revacinação com esse modelo de vacina foi eficiente contra infecções causadas pela bactéria Lactococcus garviaea, sendo uma alternativa viável para a imunização de espécies de peixes com ciclos de produção longos.

Vacinas vivas, inativadas e de DNA (menor frequência) são os principais tipos utilizados. Diversas metodologias são utilizadas para o desenvolvimento dessas vacinas, porem para a obtenção de produtos eficientes à identificação dos principais fatores de virulência dos microrganismos deve ser o ponto de partida. Esses são utilizados posteriormente como antígenos vacinais para a imunização dos animais. Porem, apesar de tal premissa, a maioria das vacinas 
utilizadas na aqüicultura tem sido desenvolvidas de forma empírica, muitas vezes por tentativa e erro. (Gudding et al., 1999).

Vacinas contra F. columnare, A. hydrophila e $S$. agalactiae tem sido descritas na literatura. A imunização de Ictalurus punctatus via imersão com vacina viva modificada contra $F$. columnare mostrou-se eficiente na indução de resposta imune, conferindo proteção aos animais quando desafiados experimentalmente (Shoemaker et al., 2007). De maneira similar, vacina viva atenuada (Vivas et al., 2004; Vivas et al., 2005) e fantasmas bacterianos (envelope celular ausente de conteúdo citoplasmático) (Chu et al., 2008) contra Aeromonas hydrophila promoveram incremento na imunidade das espécies testadas. Tilápias imunizadas com bacterina mais produto extracelular concentrado de $S$. agalactiae apresentaram proteção significativa à infecção quando comparadas aos grupos controle não vacinados (Evans et al., 2004; Pasnik et al., 2005a; Pasnisk et al., 2005b).

Atualmente, já existem vacinas comerciais contra alguns desses patógenos, porem, nenhuma delas está licenciada para uso no Brasil. Alem disso, não existem estudos da eficiência desses produtos frente a desafios com as amostras isoladas no país. Variabilidade genética e fenotípica têm sido observadas quando amostras nacionais são comparadas com amostras de referencia e isoladas de outras localidades, principalmente para F. columnare (Figueiredo et al., 2005; Olivares-Fuster et al., 2007a) e S. agalactiae (Olivares-Fuster et al., 2008). Para F. columnare tais variações se refletem na virulência e predileção por hospedeiro (Olivares-Fuster et al., 2007b; Shoemaker et al., 2008). Não existem dados, porem, é provável que as amostras de S. agalactiae e A. hydrophila apresentem comportamento similar. Tais indícios embasam a necessidade por informações sobre o perfil antigênico dos patógenos isolados no país.

O desenvolvimento de vacinas é um processo baseado na identificação de estruturas únicas presentes nas células de um determinado microrganismo, gerando imunidade quando administrada como vacina. Características como ser conservado entre diferentes isolados, induzir a formação e anticorpos específicos, conferir proteção, segurança e ser eficiente para o hospedeiro imunizado são características fundamentais para um candidato a antígeno vacinal (Chakravarti et al., 2001). A parede celular (Gram positivas) e membrana externa (Gram negativas) são as interfaces da célula bacteriana com o ambiente ou hospedeiro, no caso de bactérias patogênicas. Essas estruturas ancoram proteínas e outras substâncias essências para adesão, invasão, imunoevasão, resistência à fagocitose etc. durante os processos patogênicos, alem disso, auxiliam na manutenção da integridade celular e adaptação ao ambiente. Devido ao contato direto dessas com as células do sistema durante as infecções essas são os principais candidatos a antígenos vacinais, principalmente as proteínas (Cordwell, 2007; Harding et al., 2007). Três métodos principais são utilizados rotineiramente para identificação de antígenos na superfície das células bacterianas. $O$ primeiro é baseado na predição "in silico" da proteínas a partir do genoma do microrganismos, com o uso de algoritmos como o PSORT. Esse método é rápido, porem, necessita do genoma completo da bactéria estudada e não estima a concentração na célula. $\mathrm{O}$ segundo é realizado através da separação das substancias presentes no citoplasma dos constituintes da membrana celular por eletroforese em duas dimensões (2D) ou cromatografia 2D seguida de determinação espectrometria de massa. O terceiro método utiliza um dos supracitados para definição das proteínas encontradas na superfície celular são posteriormente avaliadas por ensaios imunológicos (anticorpos marcados ou immunoblotting) (RodriguezOrtega et al., 2006).

A eletroforese 2D é o método mais utilizado para determinação do perfil protéico de células bacterianas e produtos extracelulares de patógenos clássicos com Escherichia coli (Han e Lee, 2006). Essa técnica tem sido usada em analises imunoproteômicas para caracterização antigênica de Flavobacterium psychrophilum (Crump et al., 2001;) e identificação de potenciais antígenos vacinais para $S$. iniae (Shin et al., 2007) e F. psychrophilum (Sudheesh et al., 2007), patógenos importantes para a tilapicultura e salmonicultura mundial, respectivamente.

A partir do mapa proteômico obtido na eletroforese 2D, comparações do perfil imunogênico de diferentes isolados podem ser avaliados computacionalmente. Esse tipo metodologia tem sido aplicada para comparação de diferentes amostras e espécies bacterianas de importância médica como Burkholderia pseudomallei e Burkholderia thailandensis 
(Wongtrakoongate et al., 2007) e veterinária com $S$. aureus isolados de casos de mastite bovina (Taverna et al., 2007). A detecção de antígenos comuns em diferente isolados bacterianos possibilita o desenvolvimento de vacinas eficientes contra um maior numero de amostras.

\section{Referências Bibliográficas}

Arias, C. R. et. al. Genetic fringerprinting of Flavobacterium columnare isolates from cultured fish. J. Applied Microbiol., 97, 421-428, 2004.

Bader, J.A., Klesius, P.H., Vinitnantharat, S. Comparison of whole-cell antigens of pressure-and formalin-killed Flexibacter columnaris from channel catfish (Ictalarus punctatus). Am. J. Vet. Res., 58, 985-8, 1997.

Bader, J.A., Shotts, E.B. Identification of Flavobacterium and Flexibacter species by species-specific polymerase chain reaction primers to the $16 \mathrm{~S}$ ribosomal RNA gene. J. Aqua. Ani. Healt., 10, 311-19, 1998.

Bader, J.A., Shoemaker, C.A., Klesius, P.H. Rapid detection of columnaris disease in channel catfish (Ictalarus punctatus) with a ney species-specific 16-S rRNA gene-based PCR primer for Flavobacterium columnare. J. Microbiol. Methods, 52, 209-20, 2003.

Chacón, M. R., Soler, L., Groisman, E. A., Guarro, J., Figueras, M. J. Type III secretion system genes in clinical Aeromonas isolates. J. Clin. Microbiol., 42, 1285-7, 2004.

Chakravarti, D. N., Fiske, M. J., Fletcher, L. D., Zagursky, R. J. Application of genomics and proteomics for identification of bacterial gene products as potential vaccine candidates. Vaccine, 19,601-12, 2001.

Chu, W. H., Lu, C. P. Multiplex PCR assay for detection of pathogenic Aeromonas hydrophila. J. Fish Dis., 28, 437-41, 2005.

Chu, W., Zhuang, X., Lu, C. Generation of Aeromonas hydrophila ghosts and their evaluation as oral vaccine candidates in Carassius auratus gibelio. Wei Sheng Wu Xue Bao, 48, 202-6, 2008.

Coburn, B., Sekirov, I., Finlay, B. B. Type III secretion systems and disease. Clin. Microbiol. Rev., 20, 535 -49, 2007.

Cordwell, S. J. Technologies for bacterial surface proteomics. Cur. Opin. Microbiol., 9, 320-29, 2006.

Cornelis, G. R., Gijesem, F. V. Assembly and function of type III secretory sistems. Ann. Rev. Microbiol., 54, 735 - 74, 2000.

Darwish, A. M. et al. Identification of Flavobacterium columnare by a species-specific polymerase chain reaction and renaming of ATCC 43622 strain to Flavobacterium johnsoniae. Mol. Celul. Probes, 18, 421-427, 2004.

Decostere, A., Haesebrouck, F., Devriese, L.A. Shieh Medium Supplemented with Tobramycin for Selective Isolation of Flavobacterium columnare (Flexibacter columnaris) from Diseaded Fish. J. Clin. Microbiol., 35, 322-24, 1997.

Decostere, A. et al. Characterization of the adhesion of Flavobacterium columnare to gill tissue. J. Fish Dis., 22, 466474, 1999.

El-Sayed, A. F. M. Alternative dietary protein sources for farmed tilapia, Oreochromis spp. Aquaculture, 179, 149-68, 1999.
Evans, J. J., Klesius, P. H., Gilbert, P. M., Shoemaker, C. A., Sarawi, M. A. A. L., Landsberg, J., Duremdez, R., Markouk, A. A. L., Kenzi, S. A. L. Characterization of $\beta$-haemolitic group B Streptococcus agalactiae in cultured seabream, Sparus auratus L., and wild mullet, Liza klunzingeri (Day), in Kuwait. J. Fish Dis., 25, 505-13, 2002.

Evans, J. J., Klesius, P. H., Shoemaker, C. A. Efficacy of Streptococcus agalactiae (group B) vaccine in tilapia (Oreochromis niloticus) by intraperitoneal and bath immersion administration. Vaccine, 22, 3769-73, 2004.

FAO. State of World Aquaculture, FAO Fisheries Department. Rome, Italy. p.145, 2006.

Fasola, E., Livdahl, C., Ferrieri, P. 1993. Molecular analysis of multiple isolates of the major serotypes of group B streptococci. J. Clin. Microbiol. 31, 2616 -20, 1993.

Figueiredo, H. C. P. et al., Isolation and characterization of strains of Flavobacterium columnare from Brazil. J. Fish Dis., 28, 199-204, 2005.

Figueiredo, H. C. P. et al. Streptococcus agalactiae associado à meningoecefalite e infecção sistêmica em tilápia do Nilo (Oreochromis niloticus). Arq. Bras. Méd. Vet. e Zootec., 58, 678-680, 2006.

Figueiredo, H. C. P., LEAL, C. A. G. Columnariose: Doença da Piscicultura Moderna. Panorama da Aqüicultura, Maio, 32 37, 2007.

Figueiredo, H. C. P. et al. Estreptococose em tilápia do Nilo parte 1. Panorama da Aqüicultura, Setembro, 36 - 38, 2007a.

Figueiredo, H. C. P. et al. Estreptococose em tilápia do Nilo parte 2. Panorama da Aqüicultura, Novembro, 42 - 45, 2007b.

Fitzsimmons, K. Future trends of tilapia aquaculture in Americas. p. 252-264. In. COSTA-PIERCE, B. A.; RAKOCY, J. E. Tilapia Aquaculture in the Americas, vol.2. The World Aquaculture Society, Baton Rouge, Louisiana, USA. 2000.

Gudding, R., Lillehauga, A., Evensen, O. Recent developments in fish vaccinology. Vet. Immunol. Immunopathol., 72, 203$12,1999$.

Han, M., Lee, S. Y. The Escherichia coli Proteome: Past, Present, and Future Prospects. Microbiol. Mol. Biol. Rev., 70, 362-439, 2006.

Hirsch, D., Figueiredo, H.C.P. Identificação e resistência a antimicrobianos em espécies de Aeromonas móveis isoladas de peixes e ambientes aquáticos. Ciência e Agrotecnologia, 30 (6): 1211-1217, 2006.

Keefe, G. P., 1997. Streptococcus agalactiae mastitis: a review. Can. Vet. J., 38, 429-437.

Klesius, P. H., Evans, Joyce, J., Shoemaker, C. A. Warmwater fish vaccinology in catfish production. Animal Health Resear. Rev., 5, 305-311, 2004.

Kozinska, A. Dominant pathogenic species of mesophilic aeromonads isolated from diseased and healthy fish cultured in Poland. J. Fish Dis., 30, 293-301, 2007.

Maione, D., et al. 2005. Group B Streptococcus Vaccine by Multiple Genome Screen. Science, 309, 148-150.

Martins, E. R., Pessanha M. A., Ramirez, M., Melo-Cristino, J., Portuguese Group for the Study of Streptococcal Infections. Analysis of Group B Streptococcal Isolates from Infants and Pregnant Women in Portugal Revealing Two Lineages with Enhanced Invasiveness. J. Clin. Microbiol., 45, 3224-29, 2007.

Noga, E. J. Fish Disease: diagnosis and treatment. St Louis: Mosby-Year Book, 1996,367 p.

Olivares-Fuster, O., Shoemaker, C. A., Klesius, P. H., Arias, C. R. Molecular typing of isolates of the fish pathogen, Flavobacterium columnare, by single-strand conformation polymorphism analysis. FEMS Microbiol. Lett., 269, 63-69, 2007a. 
Olivares-Fuster, O., Baker, J. L., Terhune, J. S., Shoemaker, C. A., Klesius, P. H., Arias, C. R. Host-specific association between Flavobacterium columnare genomovars and fish species. Syst. Appl. Microbiol., 30, 624-33, $2007 \mathrm{~b}$.

Olivares-Fuster, O., Klesius, P. H., Evans, J., Arias, C. R. Molecular typing of Streptococcus agalactiae isolates from fish. J. Fish Dis., 31, 277-83, 2008.

Pasnik, D. J., Evans, J. J., Panangala, V. S., Klesius, P. H., Shelby, R. A., Shoemaker, C. A. Antigenicity of Streptococcus agalactiae extracellular products and vaccine efficacy. J. Fish Dis., 28, 205-12, 2005a.

Pasnik, D. J., Evans, J. J., Klesius, P. H. Duration of protective antibodies and correlation with survival in Nile tilapia Oreochromis niloticus following Streptococcus agalactiae vaccination. Dis. Aquat. Organ., 66,129-34, 2005b.

Pavanelli, G. C., Eiras, J. C., Takemoto, R. M. Doenças de peixes: profilaxia, diagnóstico e tratamento. Maringá: Ed. UEM, $2^{\text {a }}$ Ed., 305p., 2002

Rodrıguez-Ortega, M. J., Norais, N., Bensi, G., Liberatori, S., Capo, S., Mora, M., Scarselli, M., Doro, F., Ferrari, G., Garaguso, I., Maggi, T., Neumann, A., Covre, A., Telford, J. L., Grandi, G. Characterization and identification of vaccine candidate proteins through analysis of the group A Streptococcus surface proteome. Nat. Biotechnol., 24, 191-197, 2006.

Romalde, J. L., Luzardo-Alvarez, A., Raveloa, C., Toranzo, A. E., Blanco-Mendez, J.. Oral immunization using alginate microparticles as a useful strategy for booster vaccination against fish lactoccocosis. Aquaculture, 236 119-129, 2004.

Saavedra, M. J., Figueras, M. J., Martínez-Murcia, A. J. Updated phylogeny of the genus Aeromonas. Int. J. Syst. Evol. Microbiol., 56, 2481-7, 2006.

Sha, J., Pillai, L., Fadl, A. A. , Galindo, C. L., Erova, T. E., Chopra, A. K. The type III secretion system and cytotoxic enterotoxin alter the virulence of Aeromonas hydrophila. Infec. Immun., 73, 6446-57, 2005

Shin, G., Palaksha, K.J., Kima, Y., Nhoa, S., Kima, S., Heob, G., Park, S., Jung, T. Application of immunoproteomics in developing a Streptococcus iniae vaccine for olive flounder (Paralichthys olivaceus). J. Chromatograph. B, 849, 315-322, 2007.

Shoemaker, C. A., Klesius, P. H., Evans, J. J. Immunization of eyed channel catfish, Ictalurus punctatus, eggs with monovalent Flavobacterium columnare vaccine and bivalent $F$. columnare and Edwardsiella ictaluri vaccine. Vaccine, 25, 1126-31, 2007.

Shoemaker, C. A., Olivares-Fuster, O., Arias, C. R., Klesius, P. $\mathrm{H}$. Flavobacterium columnare genomovar influences, mortality in channel catfish (Ictalurus punctatus). Vet. Microbiol., 127, 353-9, 2008.

Sudheesh, P. S., LaFrentz, B. R., Call, D. R., Siems, W. F., LaPatra, S. E., Wiens, G. D., Cain, K. D. Identification of potential vaccine target antigens by immunoproteomic analysis of a virulent and a non-virulent strain of the fish pathogen Flavobacterium psychrophilum. Dis. Aquat. Organ., 74, 37-47, 2007.
Suomalainem, L. R.; Tiirola, M.; Valtonem, E. T. Influence of rearing conditions on Flavobacterium columnare infection of rainbow trout, Oncorhynchus mykiss (Walbaum). J. Fish Dis., 28. 271-7, 2005a.

Suomalainem, L. R.; Tiirola, M.; Valtonem, E. T. Chondroitin AC lyase is related to virulence of fish pathogenic Flavobacterium columnare. J. Fish Dis., 29, 757-763, 2005 b.

Taverna, F., Negri, A., Piccinini, R., Zecconi, A., Nonnis, S., Ronchi, S., Tedeschi, G. Characterization of cell wall associated proteins of a Staphylococcus aureus isolated from bovine mastitis case by a proteomic approach. Vet. Microbiol., 119, 240-7, 2007.

Tonheim, T. C., Bøgwald, J., Dalmo, R. A. What happens to the DNA vaccine in fish? A review of current knowledge. Fish Shellfish Immunol., aceito para publicação, Mar. 19, 2008.

Vandenberg, G. W. Oral vaccines for finfish: academic theory or commercial reality? Animal Health Res. Rev., 5, 301-304, 2004.

Vera-Calderon, L. T., Ferreira, A. C. M. Estudo da economia de escala na piscicultura em tanque-rede no Estado de São Paulo. Informações Econômicas, 34, 7-17, 2004.

Vilches, S., Urgell, C., Merino, S., Chacón, M. R., Soler, L., Castro-Escarpulli, G., Figueras, M. J., Tomás, J. M. Complete type III secretion system of a mesophilic Aeromonas hydrophila strain. Appl. Environ. Microbiol., 70, 6914-9, 2004.

Vivas, J., Carracedo, B., Riaño, J., Razquin, B. E., LópezFierro, P., Acosta, F., Naharro, G., Villena, A.J. Behavior of an Aeromonas hydrophila aroA live vaccine in water microcosms. Appl. Environ. Microbiol., 70, 2702-8, 2004.

Vivas, J., Razquin, B., López-Fierro, P., Villena, A. J. Modulation of the immune response to an Aeromonas hydrophila aroA live vaccine in rainbow trout: effect of culture media on the humoral immune response and complement consumption. Fish Shellfish Immunol., 18, 22333, 2005.

Wongtrakoongate, P., Mongkoldhumrongkul, N., Chaijan, C., Sumalee Kamchonwongpaisan, S., Tungpradabkul, S. Comparative proteomic profiles and the potential markers between Burkholderia pseudomallei and Burkholderia thailandensis. Mol. Cel. Probes, 21, 81-91, 2007.

Yu, H. B., Rao, P. S. S., Lee, H. C., Vilches, S., Merino, S., Tomas, J. M., Leung, K. Y. A type III secretion system is required for Aeromonas hydrophila $\mathrm{AH}-1$ pathogenesis. Infec. Immun., 72, $1248-56,2004$.

$\mathrm{Yu}, \mathrm{H}$. B. et. al. Identification and Characterization of Putative Virulence Genes and Genes Clusters in Aeromonas hydrophila PPD134/91. Appl. Environm. Microbiol., 71, 4469-77, 2005. 